Catherine M. Roe, $\mathrm{PhD}$ Anne M. Fagan, $\mathrm{PhD}$ Elizabeth A. Grant, PhD Jason Hassenstab, PhD

Krista L. Moulder, $\mathrm{PhD}$

Denise Maue Dreyfus, MA

Courtney L. Sutphen, BS

Tammie L.S. Benzinger, MD

Mark A. Mintun, MD

David M. Holtzman, MD

John C. Morris, MD

Correspondence to

Dr. Roe:

cathyr@wustl.edu

Supplemental data at www.neurology.org

\title{
Amyloid imaging and CSF biomarkers in predicting cognitive impairment up to 7.5 years later
}

ABSTRACT

Objectives: We compared the ability of molecular biomarkers for Alzheimer disease (AD), including amyloid imaging and CSF biomarkers $\left(A \beta_{42}, \operatorname{tau}, \operatorname{ptau}_{181}, \operatorname{tau} / A \beta_{42}, \operatorname{ptau}_{181} / A \beta_{42}\right)$, to predict time to incident cognitive impairment among cognitively normal adults aged 45 to 88 years and followed for up to 7.5 years.

Methods: Longitudinal data from Knight Alzheimer's Disease Research Center participants ( $N=$ 201) followed for a mean of 3.70 years (SD $=1.46$ years) were used. Participants with amyloid imaging and CSF collection within 1 year of a clinical assessment indicating normal cognition were eligible. Cox proportional hazards models tested whether the individual biomarkers were related to time to incident cognitive impairment. "Expanded" models were developed using the biomarkers and participant demographic variables. The predictive values of the models were compared.

Results: Abnormal levels of all biomarkers were associated with faster time to cognitive impairment, and some participants with abnormal biomarker levels remained cognitively normal for up to 6.6 years. No differences in predictive value were found between the individual biomarkers $(p>0.074)$, nor did we find differences between the expanded biomarker models $(p>0.312)$. Each expanded model better predicted incident cognitive impairment than the model containing the biomarker alone $(p<0.005)$.

Conclusions: Our results indicate that all AD biomarkers studied here predicted incident cognitive impairment, and support the hypothesis that biomarkers signal underlying AD pathology at least several years before the appearance of dementia symptoms. Neurology ${ }^{\circledR}$ 2013;80:1784-1791

\section{GLOSSARY}

$\mathbf{A} \boldsymbol{\beta}=$ amyloid- $\beta ; \mathbf{A D}=$ Alzheimer disease; $\mathbf{A D R C}=$ Alzheimer's Disease Research Center; $\mathbf{B P}=$ binding potential; $\mathbf{C D R}=$ Clinical Dementia Rating; CPE = concordance probability estimate; $\mathbf{M C B P}=$ mean cortical binding potential; PiB = Pittsburgh compound $\mathrm{B} ; \mathbf{p t a u}=$ phosphorylated tau; $\mathbf{R O I}=$ region of interest.

Biomarkers may signal underlying Alzheimer disease (AD) pathology a decade or more before the appearance of dementia symptoms. ${ }^{1,2}$ Therefore, understanding the temporal relationships between biomarker levels in cognitively normal adults, symptomatic $\mathrm{AD}$ (i.e., incident $\mathrm{AD}$ ), and factors that modify those relationships is imperative. ${ }^{3}$ The National Institute on Aging/ Alzheimer's Association workgroup urges that the factors that best predict progression from normal cognition to cognitive impairment and dementia due to AD need to be determined. ${ }^{3}$

The most well-studied and promising molecular biomarkers of $\mathrm{AD}$ are those that reflect the presence of the signature lesions of $\mathrm{AD}$ : plaques, which comprise the amyloid- $\beta$ (A $\beta$ ) protein, and tangles, which comprise the tau protein. Both amyloid imaging, ${ }^{4}$ used to identify fibrillar $A \beta$ plaques, and CSF biomarkers, ${ }^{5}$ which reflect soluble $A \beta$, tau, and phosphorylated tau (ptau), predict incident AD. ${ }^{6-8}$ However, until now, directly comparing the predictive ability of amyloid imaging with CSF biomarkers was difficult, given the recent development of amyloid imaging ${ }^{4}$ and attendant short follow-up times, the lengthy hypothesized time between the appearance of $\mathrm{AD}$

\footnotetext{
From the Knight Alzheimer's Disease Research Center (C.M.R., A.M.F., E.A.G., J.H., K.L.M., D.M.D., T.L.S.B., D.M.H., J.C.M.), Hope Center for Neurological Disorders (A.M.F., D.M.H.), and Departments of Neurology (C.M.R., A.M.F., K.L.M., C.L.S., D.M.H., J.C.M.), Psychology (J.H., D.M.D.), Radiology (T.L.S.B., M.A.M.), Pathology and Immunology (J.C.M.), Physical Therapy (J.C.M.), Occupational Therapy (J.C.M.), and Neurosurgery (T.L.S.B.), and the Division of Biostatistics (E.A.G.), Washington University School of Medicine, St. Louis, MO; and Avid Radiopharmaceuticals (M.A.M.), Philadelphia, PA.

Go to Neurology.org for full disclosures. Funding information and disclosures deemed relevant by the authors, if any, are provided at the end of the article.
} 
pathology and dementia symptoms, and participant cohorts lacking measurement of both biomarker types.

Our primary goal is to compare individual molecular biomarkers of $\mathrm{AD}$ in cognitively normal persons in relation to incident cognitive dysfunction as a necessary first step for future individual-level prediction. Consistent with this goal, we examined whether incorporating demographic information strengthened the predictive power of the biomarker models.

METHODS Participants. We used archival data that were collected prospectively from participants enrolled in longitudinal studies at the Knight Alzheimer's Disease Research Center (ADRC). Recruitment procedures have been reported. ${ }^{9}$ In brief, participants are recruited through word-of-mouth, advertisements, and community events for annual clinical assessments. Participants are representative of older adult cohorts used in research. Individuals with comorbid disorders are included ${ }^{10}$ unless their health conditions may interfere with longitudinal follow-up (e.g., metastatic cancer).

Standard protocol approvals, registrations, and patient consents. Study protocols were approved by the Washington University Medical Center Human Subjects Committee, and written informed consent was obtained from all participants.

Clinical assessment. Annual clinical and neuropsychological assessments take place at the Knight ADRC. A Clinical Dementia Rating $^{11,12}$ (CDR) is derived by experienced clinicians who synthesize information obtained from interviews with the participant and separately with an informant who knows the participant well, and the neurologic examination. The CDR is reliable. ${ }^{13}$ The clinician's judgment about the presence of dementia is based on the principle of intraindividual change where the individual is used as his or her own control. The CDR is derived in accordance with a standard scoring algorithm: $0=$ no dementia, $0.5=$ very mild, $1=$ mild, $2=$ moderate, and $3=$ severe dementia. $\mathrm{ACDR}$ $\geq 0.5$ indicates clinically significant cognitive impairment. For participants who meet this criterion, a clinical diagnosis indicating the suspected etiology of the impairment is assigned. A diagnosis of $\mathrm{AD}$ dementia is based on evidence that the participant has experienced the gradual onset and progression of memory and other cognitive problems that represent a change from a previous higher level of functioning, and that interfere with usual activities at home and in the community. Neuropathologic confirmation of the clinical diagnosis of $\mathrm{AD}$ dementia is found for $93 \%$ of cases. ${ }^{14}$

Pittsburgh compound B uptake measurement. PET fibrillar $A \beta$ imaging was performed and processed as previously described..$^{14}$ In brief, a 60-minute dynamic scan was acquired after injection of 12 to $15 \mathrm{mCI}$ of $\left[{ }^{11} \mathrm{C}\right]$ Pittsburgh compound B (PiB). Reconstructed PET frames were corrected for motion, summed, and coregistered to an anatomical MRI performed in a separate imaging session. Three-dimensional regions of interest (ROIs) were created yielding regional time-activity curves. ${ }^{14}$ Using the cerebellum ROI data as the reference tissue input function, a time activity curve for each ROI is analyzed for specific $\mathrm{PiB}$ binding. The slope of each curve reflects the tracer distribution volume in the tissue of interest relative to the input function. ${ }^{14} \mathrm{~A}$ binding potential (BP) value reflecting the ROI binding value proportional to the number of binding sites for each ROI is calculated using the equation $\mathrm{BP}=$ distribution volume -1 . The mean cortical BP (MCBP) is obtained by taking the mean of the BPs from brain regions known to have high uptake among participants with $\mathrm{AD}$ dementia: the prefrontal cortex, gyrus rectus, lateral temporal cortex, and precuneus. ${ }^{14}$

CSF measurement. Experienced neurologists used a 22-gauge Sprotte spinal needle to collect 20 to $30 \mathrm{~mL}$ of CSF via lumbar puncture at 8:00 AM after an overnight fast. CSF samples are gently inverted to avoid possible gradient effects, centrifuged at low speed to pellet any cellular elements, and frozen at $-84^{\circ} \mathrm{C}^{15}$ after aliquoting $(0.5 \mathrm{~mL})$ into polypropylene tubes. Levels of $A \beta_{42}$, total tau, and ptau $_{181}$ were measured using ELISA (INNOTEST; Innogenetics, Ghent, Belgium). Samples were analyzed in batches of 40 upon receipt. CSF levels of $A \beta_{42}$ have consistently been reported to be reduced in $\mathrm{AD}$, whereas levels of tau and ptau (and the ratios of $\left.\operatorname{tau}[s] / A \beta_{42}\right)$ are increased. ${ }^{16}$

Inclusion criteria. Data used were from cognitively normal participants (CDR 0) who 1) underwent both PET-PiB imaging and donated CSF within 1 year of clinical assessment, 2) had at least 1 additional assessment after the baseline assessment, and 3) were aged 45 years or older. To compare biomarker models across the same individuals, included participants were also required to have nonmissing data on all variables of interest.

Statistical analyses. Using separate Cox proportional hazards models, we first tested whether each of the individual biomarker variables (MCBP, $A \beta_{42}$, tau, $p \operatorname{ptau}_{181}, \operatorname{tau} / A \beta_{42}, p \operatorname{ptau}_{181} / A \beta_{42}$ ) was related to time to incident cognitive abnormality, defined by first $\mathrm{CDR}>0$. To ascertain whether combining biomarkers with participant demographic information would improve longitudinal prediction of cognitive impairment, "expanded" Cox proportional hazards models were developed using the stepwise selection method with 0.05 used as both the probability to enter, and to exit, the model. Candidate variables for stepwise selection for each model were the biomarker variable, age, sex, minority vs white race, education, and the presence of at least 1 APOE $\varepsilon 4$ allele (APOE4).

A concordance probability estimate $(\mathrm{CPE})^{17}$ reflecting the accuracy of the predictive Cox proportional hazards model was calculated for all models. The CPEs of the predictive biomarker models were statistically compared with each other to determine which models had better predictive accuracy.

In the Cox models, data from participants who died, who did not return for follow-up, or who did not develop cognitive impairment over the follow-up period were statistically censored at the date of the most recent clinical assessment.

We also examined whether each of the biomarkers predicted cognitive decline on 5 psychometric tests common to all Knight ADRC longitudinal protocols (Animal Naming, ${ }^{18}$ Trailmaking A test, ${ }^{19}$ Trailmaking B test, ${ }^{19}$ Selective Reminding Test-Free Recall subtest, ${ }^{20}$ and the Mini-Mental State Examination ${ }^{21}$ ) using linear mixed models, which included the intercept and slope terms as random effects. Similar analyses were conducted for a global composite psychometric score, ${ }^{22}$ which was available for a subset of participants who had the same psychometric test battery. The linear mixed models were adjusted for the same demographic variables selected by the stepwise procedure in the expanded Cox proportional hazards model containing the biomarker of interest.

We repeated these analyses treating the biomarker values as dichotomous. Abnormal biomarker values are referred to as positive $(+)$ and normal values are referred to as negative $(-)$ subsequently in this report and were based on cutoffs used previously ( $\geq 0.18$ for MCBP, ${ }^{23}<500 \mathrm{pg} / \mathrm{mL}$ for $\mathrm{A}_{42},{ }^{24}>440 \mathrm{pg} / \mathrm{mL}$ for tau, ${ }^{24}>78 \mathrm{pg} / \mathrm{mL}$ for $\operatorname{ptau}_{181},{ }^{24}>0.94$ for tau $/ A \beta_{42},{ }^{24}$ and $>0.15$ for $\left.\mathrm{ptau}_{181} / \mathrm{A}_{42}{ }^{24}\right)$. 
RESULTS Available CSF data spanned August 1998 to July 2010, and MCBP data spanned April 2004 to March 2011. A total of 201 individuals aged 45.3 to 88.6 years met all inclusion criteria (table 1 ). Their clinical assessment dates ranged from February 2004 to January 2012. Data from some of these participants have been used in other studies. ${ }^{25,26}$ Table e-1 (on the Neurology ${ }^{\circledR}$ Web site at www.neurology.org) shows the correlations among the biomarkers and continuous demographic variables. Because we were surprised to find here, and previously, ${ }^{27}$ that fibrillar amyloid (MCBP) values were more highly correlated with the tau/A $\beta_{42}$ and ptau/A $\beta_{42}$ values than with the CSF measures of $A \beta_{42}$ or tau alone, we graphed the relationships among $A \beta_{42}$, tau, and MCBP. As shown in figure 1 , larger MCBP values tend to occur in the presence of both smaller $A \beta_{42}$ values and larger tau values. This impression was confirmed by a general linear model, which showed that $A \beta_{42}$ and tau interact to predict MCBP $(p<0.001)$ after adjustment for the demographic variables. However, $\mathrm{A} \beta_{42}$ and MCBP do not interact to predict tau values $(p=0.993$, figure $\mathrm{e}-1)$, nor do tau and MCBP values

\begin{tabular}{|c|c|c|}
\hline Demographic & $N=201)$ & \\
\hline & No. or mean & $\%$ or SD \\
\hline Age, y & 67.8 & 9.1 \\
\hline Women & 128 & $63.7 \%$ \\
\hline Minority race & 15 & $7.5 \%$ \\
\hline Education, y & 15.6 & 2.6 \\
\hline $\begin{array}{l}\text { Mini-Mental State } \\
\text { Examination score }\end{array}$ & 29.0 & 1.3 \\
\hline $\begin{array}{l}\text { Mean cortical binding } \\
\text { potential for PiB }\end{array}$ & 0.12 & 0.24 \\
\hline $\mathrm{PiB}+{ }^{\mathrm{b}}$ & 41 & $20.4 \%$ \\
\hline$A \beta_{42}, p g / m L$ & 630.35 & 251.58 \\
\hline tau, $\mathrm{pg} / \mathrm{mL}$ & 289.36 & 159.52 \\
\hline $\mathrm{ptau}_{181}, \mathrm{pg} / \mathrm{mL}$ & 53.82 & 24.93 \\
\hline tau $/ A \beta_{42}$ ratio & 0.58 & 0.52 \\
\hline $\operatorname{ptau}_{181} / \mathrm{A} \beta_{42}$ ratio & 0.11 & 0.08 \\
\hline $\begin{array}{l}\text { Interval between clinical } \\
\text { assessment and PET PiB, y }\end{array}$ & 0.40 & 0.26 \\
\hline $\begin{array}{l}\text { Interval between clinical } \\
\text { assessment and LP, y }\end{array}$ & 0.30 & 0.21 \\
\hline $\begin{array}{l}\text { Interval between PET PiB } \\
\text { and LP, y }\end{array}$ & 0.33 & 0.35 \\
\hline Follow-up time, y & 3.70 & 1.46 \\
\hline
\end{tabular}

Abbreviations: $A \beta=$ amyloid $-\beta$; $L P=$ lumbar puncture; $\mathrm{PiB}=$ Pittsburgh compound $\mathrm{B} ;$ ptau = phosphorylated tau. ${ }^{\text {a }}$ Mini-Mental State Examination scores range from $O$ (worst performance) to 30 (best performance). In a population-based US sample, and among individuals aged 65 to 69 years with 13 or more years of education, the median Mini-Mental State Examination score is 29.40

${ }^{\mathrm{b}} \mathrm{PiB}+$ indicates mean cortical binding potential $>0.18$. interact to predict $A \beta_{42}$ values ( $p=0.793$, figure e-2). Similar results were found for analyses testing ptau rather than tau.

As reported previously, ${ }^{23} \mathrm{MCBP}(p<0.001)$ and $A \beta_{42}(p<0.001)$, but not tau and ptau $(p>0.258)$, were associated with number of APOE4 alleles in this sample. Mean MCBP values were 0.078, 0.157, and 0.424, and $A \beta_{42}$ values were 666.9, 586.7, and $350.2 \mathrm{pg} / \mathrm{mL}$, for individuals with 0,1 , and 2 APOE 4 alleles, respectively. Biomarker values were unrelated to sex and race $(p>0.190)$.

Of the included participants, 28 (13.9\%) developed incident cognitive impairment (defined as CDR $\geq 0.5)$ over a mean follow-up period of 3.7 years (range $0.96-7.53$ years). Mean CDR sum of boxes score at the time of first CDR $>0$ was 1.42 $(\mathrm{SD}=0.89)$. Of the 22 participants with at least 1 additional assessment after their first CDR $>0,7$ had CDR 0 and 15 had CDR $>0$ at their most recent assessment.

In the Cox models testing the biomarkers alone, abnormal values for all biomarkers were associated with a faster time to cognitive impairment (table 2). Calculation of $\mathrm{CPE}$ values reflects the predictive accuracy of a model containing censored data, and thus, is conceptually similar to using a receiver operating characteristic curve to assess the predictive value of a model predicting a binary outcome..$^{28}$ As with the area under the receiver operating characteristic curve, larger CPE values reflect more accurate predictive accuracy. ${ }^{28}$ No differences in predictive value were found between the CPEs yielded by the individual biomarkers $(p>0.077)$ treated continuously. When treated dichotomously, $A \beta_{42}$ was a better predictor than tau $(p=0.019)$, but there were no other differences between the biomarkers ( $p>0.067)$.

In the stepwise models, older participant age, male sex, and minority race indicated faster time to cognitive impairment in every model (table 2). APOE4 also entered the models testing tau and ptau alone, but not models including terms reflecting $\mathrm{PiB}$ uptake or $\mathrm{A} \beta_{42}$. Once age, sex, race, and APOE4 entered the model testing ptau as a continuous variable, the ptau effect was marginally significant $(p=0.057)$. The biomarker term remained a significant predictor in the expanded models for all other biomarkers, and for ptau treated as a dichotomous variable. For all biomarkers, the expanded model resulted in better prediction of time to incident cognitive impairment than the model containing the biomarker alone $(p<$ 0.007), but the predictive values of the expanded models did not differ from each other $(p>0.281)$.

To better understand the nature of the tau/A $\beta_{42}$ and ptau $/ A \beta_{42}$ ratios and their relationship with incident cognitive impairment, we constructed a stratified variable reflecting the 4 possible combinations of 
Figure 1 Bubble plot illustrating the association of CSF $A \beta_{42}$ and CSF tau with fibrillar $A \beta$ binding imaged using PET with Pittsburgh compound $\mathrm{B}$ (PiB) among cognitively normal adults

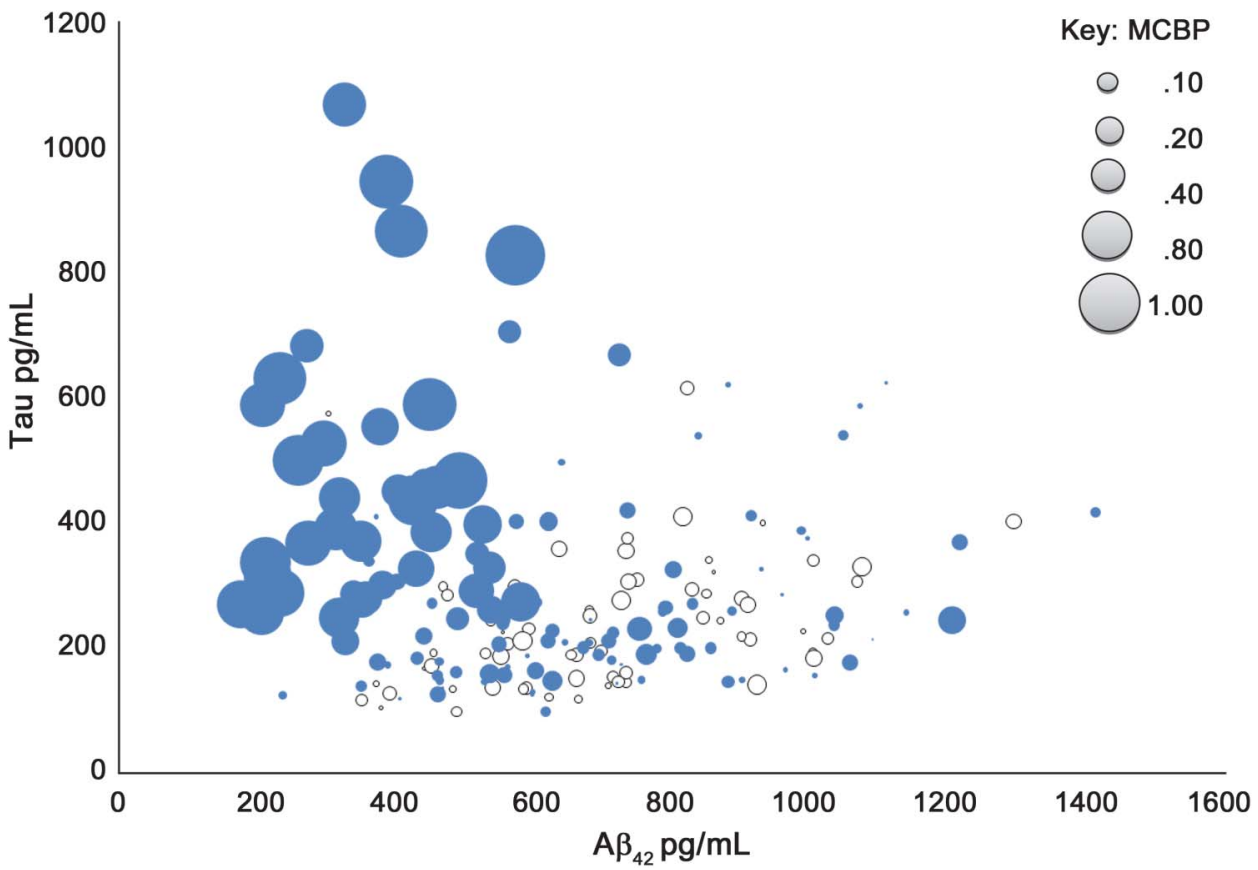

The area of the bubbles reflects the mean cortical binding potential (MCBP) for PiB value. Clear bubbles represent negative MCBP values (i.e., essentially no PiB binding).

\begin{tabular}{|c|c|c|c|c|c|c|c|c|c|c|c|c|c|}
\hline \multirow[t]{4}{*}{ Table 2} & e values & of each & h biomark & er alone & vs expa & anded $n$ & nodels ( $\mathrm{N}$ & $\mathrm{V}=201)$ & & & & & \\
\hline & \multicolumn{6}{|c|}{ Models testing the biomarkers alone } & \multicolumn{6}{|c|}{ Expanded models } & \multirow{3}{*}{$\begin{array}{l}p \text { Value testing } \\
\text { difference between CPEs }\end{array}$} \\
\hline & \multirow[b]{2}{*}{$H R^{a}$} & \multicolumn{2}{|c|}{$95 \% \mathrm{Cl}$} & \multirow[b]{2}{*}{$p$ Value } & \multirow[b]{2}{*}{ CPE } & \multirow[b]{2}{*}{ SEM } & \multirow[b]{2}{*}{$\mathrm{HR}^{\mathrm{a}}$} & \multicolumn{2}{|l|}{$95 \% \mathrm{Cl}$} & \multirow[b]{2}{*}{$p$ Value } & \multirow[b]{2}{*}{ CPE } & \multirow[b]{2}{*}{ SEM } & \\
\hline & & Lower & Upper & & & & & Lower & Upper & & & & \\
\hline \multicolumn{14}{|c|}{ Biomarkers treated as continuous variables } \\
\hline МСBP & 10.36 & 3.34 & 32.06 & $<0.001$ & 0.614 & 0.025 & 4.82 & 1.68 & 13.84 & 0.0035 & 0.783 & 0.030 & $<0.001$ \\
\hline$A \beta_{42}, \mathrm{pg} / \mathrm{mL}$ & 0.997 & 0.995 & 0.999 & 0.002 & 0.676 & 0.048 & 0.998 & 0.996 & 0.999 & 0.0066 & 0.794 & 0.029 & 0.007 \\
\hline tau, pg/mL & 1.003 & 1.001 & 1.004 & $<0.001$ & 0.605 & 0.028 & 1.002 & 1.0001 & 1.004 & 0.0384 & 0.792 & 0.030 & $<0.001$ \\
\hline $\mathrm{ptau}_{181}, \mathrm{pg} / \mathrm{mL}$ & 1.02 & 1.01 & 1.03 & $<0.001$ & 0.606 & 0.029 & 1.01 & 0.9997 & 1.02 & 0.0571 & 0.793 & 0.030 & $<0.001$ \\
\hline tau/A $\beta_{42}$ ratio & 2.98 & 2.03 & 4.36 & $<0.001$ & 0.612 & 0.019 & 2.51 & 1.63 & 3.85 & $<0.0001$ & 0.784 & 0.030 & $<0.001$ \\
\hline $\operatorname{ptau}_{181} / \mathrm{A} \beta_{42}$ ratio & 665.30 & 61.25 & 7226.68 & $<0.001$ & 0.612 & 0.021 & 330.00 & 22.14 & 4919.22 & $<0.0001$ & 0.790 & 0.030 & $<0.001$ \\
\hline \multicolumn{14}{|c|}{ Biomarkers treated as dichotomous variables } \\
\hline MCBP+ & 3.68 & 1.74 & 7.81 & $<0.001$ & 0.594 & 0.023 & 2.57 & 1.20 & 5.51 & 0.016 & 0.786 & 0.031 & $<0.001$ \\
\hline$A \beta_{42}+$ & 3.80 & 1.77 & 8.16 & $<0.001$ & 0.633 & 0.030 & 2.95 & 1.35 & 6.44 & 0.007 & 0.789 & 0.030 & $<0.001$ \\
\hline tau+ & 3.48 & 1.57 & 7.71 & 0.002 & 0.563 & 0.019 & 2.55 & 1.10 & 5.90 & 0.029 & 0.792 & 0.030 & $<0.001$ \\
\hline $\operatorname{ptau}_{181}+$ & 4.54 & 2.12 & 9.70 & $<0.001$ & 0.579 & 0.018 & 2.64 & 1.20 & 5.82 & 0.016 & 0.767 & 0.031 & $<0.001$ \\
\hline tau/A $\beta_{42}+$ & 5.78 & 2.73 & 12.23 & $<0.001$ & 0.594 & 0.018 & 4.06 & 1.89 & 8.73 & $<0.001$ & 0.787 & 0.029 & $<0.001$ \\
\hline $\operatorname{ptau}_{181} / A \beta_{42}+$ & 4.38 & 2.07 & 9.23 & $<0.001$ & 0.599 & 0.021 & 2.83 & 1.32 & 6.07 & 0.007 & 0.782 & 0.036 & $<0.001$ \\
\hline
\end{tabular}

Abbreviations: $\mathrm{A} \beta=$ amyloid $-\beta ; \mathrm{Cl}=$ confidence interval; $\mathrm{CPE}=$ concordance probability estimate; $\mathrm{HR}=$ hazard ratio; $\mathrm{MCBP}=$ mean cortical binding potential; $\mathrm{PiB}=$ Pittsburgh compound $\mathrm{B} ; \mathrm{ptau}=$ phosphorylated tau.

a The unit of measurement is important in the appropriate interpretation of HRs for continuous variables. For example, the HR of 10.10 for MCBP listed in the first column indicates that the HR is increased by $910 \%$ for each unit increase in MCBP. In this study, MCBP ranged from a low of -0.10 to a high of +1.05 . 
+ and - tau and $A \beta_{42}$ values. Individuals with abnormal levels of both tau and $A \beta_{42}$ progress most rapidly to cognitive impairment relative to the other combinations (figure $2 \mathrm{~A}$, table e-2). Similar results were found when the ptau/A $\beta_{42}$ variable was examined (figure $2 \mathrm{~B}$, table e-2).

Table e- 3 shows the results of testing the longitudinal change in psychometric test scores as a function of each biomarker. Abnormal values of all biomarkers were associated with declining cognition on at least one of the individual psychometric tests $(p<0.042)$.

As illustrated in figure 3, some individuals with abnormal biomarker levels remained cognitively normal several years after biomarker measurement. For example, of $\mathrm{PiB}+$ individuals, 5 of 8 (63\%) of those followed at least 4 years, 3 of 4 (75\%) of those

Figure 2 Survival curves reflecting time to cognitive impairment for the 4 possible combinations of positive and negative (A) CSF tau and $A \beta_{42}$ status, and (B) CSF ptau 181 and $A \beta_{42}$ status

A

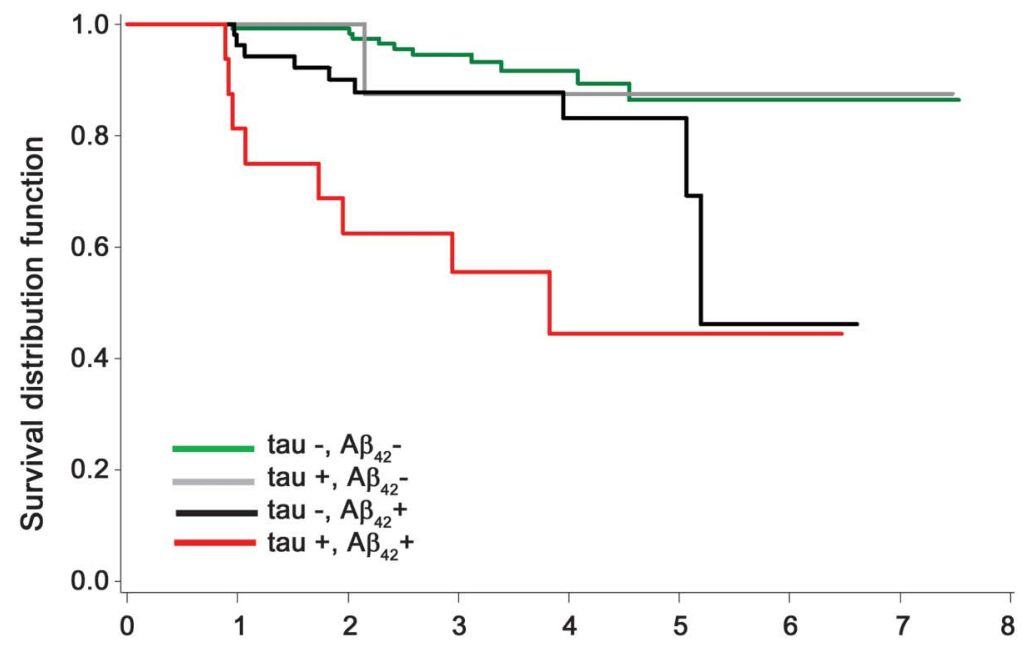

B

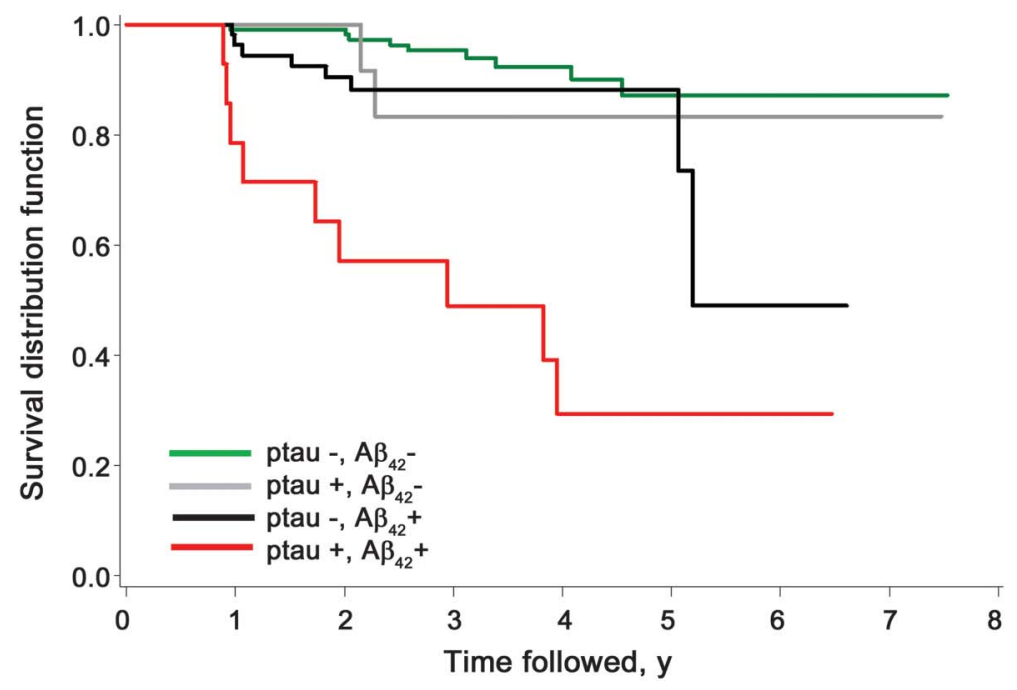

followed at least 5 years, and 2 of $3(67 \%)$ of those followed at least 6 years remained cognitively normal.

DISCUSSION We found that all molecular biomarker variables examined predicted time from cognitive normality to incident cognitive impairment. Hence, $\mathrm{AD}$ biomarkers in cognitively normal persons designate a preclinical stage of $\mathrm{AD}$ that is marked by greater risk for developing cognitive impairment due to $\mathrm{AD}$. There were no differences in the predictive ability of the biomarkers. These findings, using a larger sample size $(N=201)$, a longer range of follow-up times (up to 7.5 years), and measurement of CSF and amyloid imaging biomarkers within the same individuals at approximately the same time, extend our previous work that showed that both CSF biomarkers ${ }^{6,7}$ and amyloid imaging ${ }^{25}$ predict incident cognitive impairment.

Combining the biomarker values with information regarding certain participant characteristics resulted in better prediction of incident cognitive impairment for every biomarker. Our sample included adults aged 45 to 65 years, because previous work indicates that abnormal CSF A $\beta_{42}$ levels can occur as early as the mid- $40 \mathrm{~s},{ }^{23}$ consistent with data from families with autosomal dominant $\mathrm{AD}$ mutations suggesting that abnormal levels of the biomarkers studied here may occur 25 to 30 years before the appearance of dementia symptoms. ${ }^{29}$ The present results support the hypothesis that both abnormal biomarker levels and older age are important factors in determining time to incident $\mathrm{AD}$ (e.g., see figure 3).

Moreover, our examination of the relationships among the biomarkers themselves indicated that CSF $A \beta_{42}$ and tau interact to predict PiB uptake values, such that individuals with abnormal $\mathrm{PiB}$ uptake values usually have abnormal levels of both CSF $A \beta_{42}$ and tau, rather than either alone. The pattern of results found among these biomarkers implies that abnormal changes in both $A \beta_{42}$ and tau occur before, or coincide with, substantial deposition of fibrillar amyloid. This result is consistent with data from autosomal dominant $\mathrm{AD}$ families suggesting that changes in CSF tau and fibrillar amyloid deposition lag those of CSF $A \beta_{42} \cdot{ }^{29}$

Minority participants and men developed cognitive impairment more rapidly compared with white participants and women, after adjustment for biomarker levels. The majority $(80 \%)$ of our minority participants were African American. It has previously been established that African Americans have a higher incidence rate of $\mathrm{AD}$ compared with Caucasians. ${ }^{30}$ Because biomarker levels were included in the models, the faster time to cognitive impairment for minority participants cannot be a function of differences across the groups in average biomarker values. This is an important area for 


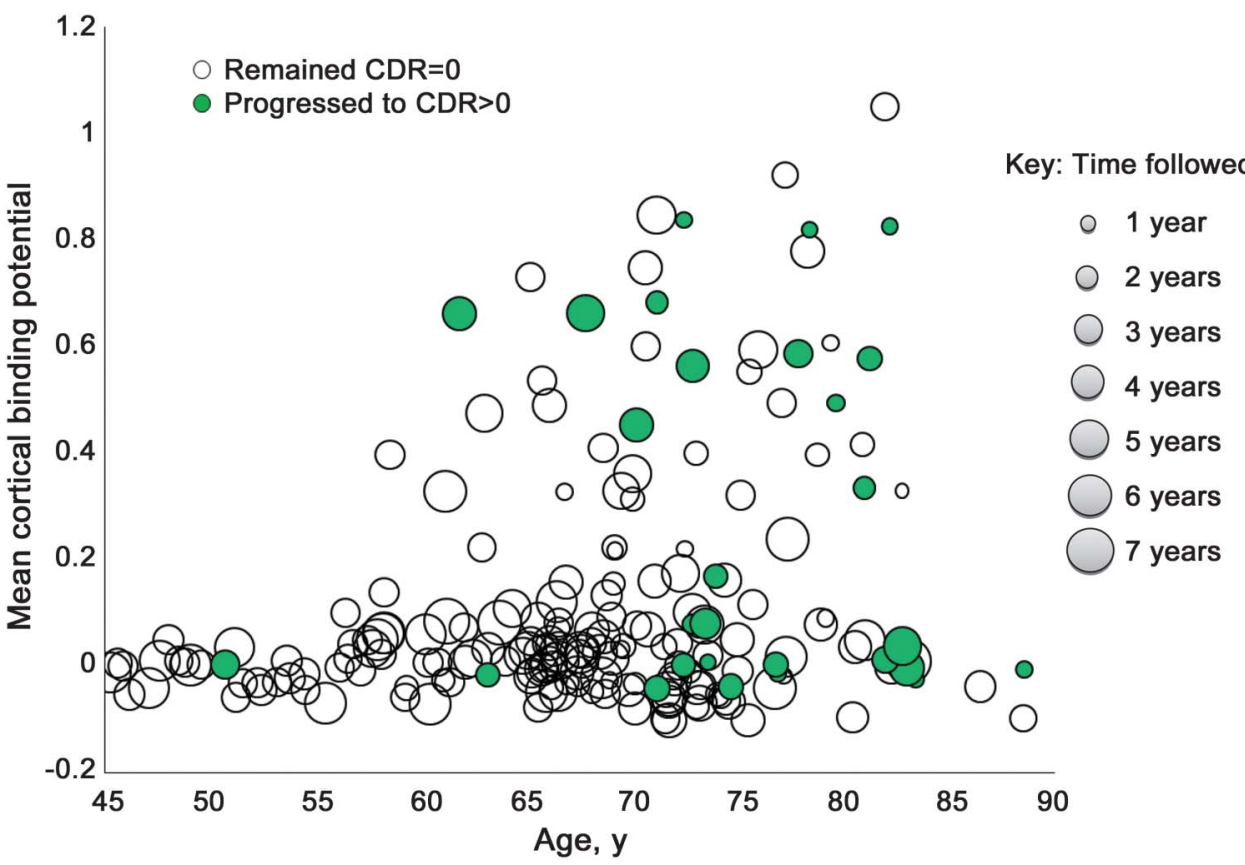

The area of the bubbles reflects time to CDR $>0$ for those who progressed, and follow-up time for those who maintained $\mathrm{CDR}=0$ during follow-up.

future research. Consistent with our results, a recent study suggests that the incidence of mild cognitive impairment is higher among men than women. ${ }^{31}$ However, other studies suggest either no gender difference in incident $\mathrm{AD}$ dementia ${ }^{32}$ or a higher incident dementia rate for women compared with men..$^{33}$ Autopsy data suggest that women are more likely to show dementia symptomatology in the presence of $\mathrm{AD}$ pathology, ${ }^{34}$ whereas our results, using biomarkers as indicators of underlying $\mathrm{AD}$ pathology, suggest the opposite.

APOE 4 aided in prediction of cognitive impairment in models testing tau and ptau, but not models testing $A \beta_{42}$, either alone or as part of the "ratio" biomarker values (i.e., tau/A $\beta_{42}$ and ptau/A $\beta_{42}$ ). Levels of $A \beta_{42}$, but not levels of tau and ptau, are associated with $A P O E$ genotypes among cognitively normal individuals. ${ }^{23}$ Therefore, APOE genotype and $A \beta_{42}$ may provide somewhat redundant information, and once $A \beta_{42}$ is included in a model, $A P O E$ genotype does not add any predictive value.

As in the models testing each biomarker alone, the expanded models including participant characteristics did not differ from each other in longitudinal predictive ability. Although much more research needs to be done in comparing imaging and CSF biomarkers, these results imply that decisions about whether imaging or CSF biomarkers are obtained to determine preclinical $\mathrm{AD}$ may eventually rely more on factors such as acceptability of the procedure to the individual and physician, the cost of the assays, and availability.

Although nearly all biomarkers were related to change in the global cognitive functioning composite score with time, not all of the individual tests that make up the composite showed significant associations. This may be attributable to the regional distribution of neuropathology in the preclinical stages of $\mathrm{AD}$. Psychometric tests that rely on integrity of regions in the limbic system and the precuneus, such as episodic memory tasks and verbal fluency tasks, are regions that also show increased amyloid deposition, ${ }^{35}$ early structural changes, ${ }^{36}$ and reduced functional connectivity, even in individuals without clinical symptoms of dementia. ${ }^{37}$

Some individuals developed cognitive impairment but had normal baseline biomarker values. There are several possible explanations for this result. First, participants may have been misdiagnosed with cognitive impairment. Second, participants may be cognitively impaired due to a non-AD mechanism, or they developed abnormal biomarker levels postbaseline. Third, the biomarkers captured here may not reflect all pathology relevant to symptomatic $\mathrm{AD}$. For example, amyloid tracers that bind strongly to fibrillar $A \beta$ plaques may be unable to detect $\mathrm{AD}$ characterized predominantly by diffuse $A \beta$ plaques. ${ }^{38}$

Likewise, some participants had abnormal biomarker levels, but did not develop cognitive impairment during the follow-up period. As noted above, biomarker abnormalities may occur 25 to 30 years 
before the appearance of dementia symptoms..$^{29}$ Additionally, cognitive and brain reserve may have important roles in determining the time that individuals with abnormal biomarker levels will remain cognitively normal. ${ }^{39}$

Finally, our results support the hypothesis that molecular biomarkers of $\mathrm{AD}$ signal underlying $\mathrm{AD}$ pathology a decade or more before the appearance of cognitive symptoms, ${ }^{1,2}$ as we found that the majority of individuals with abnormal biomarker values followed 4 to 7 years remained cognitively normal.

Limitations of our study include the use of a convenience sample, so the extent to which these results are generalizable to the larger population is unknown. Some statistically significant differences reported here may be due to chance because we conducted multiple statistical tests. With decreasing $p$ values, the likelihood that a difference is due to chance also decreases.

We find that $\mathrm{AD}$ biomarker profiles are associated with incident cognitive impairment, and hence, can be used to identify cognitively normal individuals at much higher risk of symptomatic $\mathrm{AD}$ for secondary prevention clinical trials. However, our results suggest that accurate prediction of future symptomatic $\mathrm{AD}$ for a specific individual will not depend on molecular biomarkers alone, but rather, will incorporate characteristics of the individual in making the prediction.

\section{AUTHOR CONTRIBUTIONS}

Dr. Roe: study concept and design, data analysis and interpretation, drafting and critical revision of manuscript. Dr. Fagan: data acquisition, analysis and interpretation, critical revision of manuscript, study supervision. Dr. Grant: data acquisition, analysis and interpretation, critical revision of manuscript. Dr. Hassenstab: data analysis and interpretation, critical revision of manuscript. Dr. Moulder: drafting and critical revision of manuscript. Ms. Maue Dreyfus: data acquisition, analysis and interpretation, critical revision of manuscript. Ms. Sutphen: data analysis and interpretation, critical revision of manuscript. Dr. Benzinger and Dr. Mintun: data acquisition, analysis and interpretation, critical revision of manuscript. Dr. Holtzman: data analysis and interpretation, critical revision of manuscript. Dr. Morris: data acquisition, analysis and interpretation, critical revision of manuscript, study supervision.

\section{ACKNOWLEDGMENT}

The authors thank the participants, investigators, and staff of the Knight ADRC Clinical (participant assessments) and Genetics Cores (genotyping), the investigators and staff of the Biomarker Core for the Adult Children Study (P01 AG026276) for CSF analytes, and the investigators and staff of the Imaging Core of the Healthy Aging and Senile Dementia study (P01AG03991) for amyloid imaging.

\section{STUDY FUNDING}

Funding for this study was provided by the Longer Life Foundation, the National Institute of Neurological Disorders and Stroke (P30 NS057105); National Institute on Aging (P50 AG005681, P01 AG003991, and P01 AG026276); Fred Simmons and Olga Mohan, and the Charles and Joanne Knight Alzheimer's Research Initiative of the Washington University Knight Alzheimer's Disease Research Center.

\section{DISCLOSURE}

C. Roe reports no disclosures. A. Fagan is supported by grants from the National Institute of Aging of the NIH (P01 AG03991, P01 AG026276, and U01 AG032438) and the Hope Center for Neurological Disorders, and is a member of the Alzheimer's Disease CSF Biomarker Development Advisory Board for Roche and the US Alzheimer's Disease Advisory Board for Lilly USA. No conflict of interest exists. E. Grant, J. Hassenstab, K. Moulder, D. Maue Dreyfus, and C. Sutphen report no disclosures. T. Benzinger has received research grants from Avid Radiopharmaceuticals and serves on an Advisory Board for Eli Lilly. M. Mintun is employed by Avid Radiopharmaceuticals. D. Holtzman receives research grants to his laboratory from Eli Lilly, AstraZeneca, and Pfizer. He is on the scientific advisory board of Pfizer and C2N Diagnostics. He has consulted for BristolMyers Squibb. He is a cofounder of C2N Diagnostics. J. C. Morris reports disclosures: Neither Dr. Morris nor his family owns stock or has equity interest (outside of mutual funds or other externally directed accounts) in any pharmaceutical or biotechnology company. Dr. Morris has participated or is currently participating in clinical trials of antidementia drugs sponsored by the following companies: Janssen Immunotherapy, Eli Lilly and Company, and Pfizer. Dr. Morris has served as a consultant for the following companies: Eisai, Esteve, Janssen Alzheimer Immunotherapy Program/Elan, GlaxoSmithKline, Novartis, Otsuka Pharmaceuticals, and Pfizer/Wyeth. Go to Neurology.org for full disclosures.

Received October 2, 2012. Accepted in final form January 29, 2013.

\section{REFERENCES}

1. Fagan AM, Head D, Shah AR, et al. Decreased cerebrospinal fluid $A \beta 42$ correlates with brain atrophy in cognitively normal elderly. Ann Neurol 2009;65:176-183.

2. Jack CR Jr, Knopman DS, Jagust WJ, et al. Hypothetical model of dynamic biomarkers of the Alzheimer's pathological cascade. Lancet Neurol 2010;9:119-128.

3. Sperling RA, Aisen PS, Beckett LA, et al. Toward defining the preclinical stages of Alzheimer's disease: recommendations from the National Institute on Aging-Alzheimer's Association workgroups on diagnostic guidelines for Alzheimer's disease. Alzheimers Dement 2011;7:280-292.

4. Klunk WE, Engler H, Nordberg A, et al. Imaging brain amyloid in Alzheimer's disease with Pittsburgh Compound-B. Ann Neurol 2004;55:306-319.

5. Craig-Schapiro R, Fagan AM, Holtzman DM. Biomarkers of Alzheimer's disease. Neurobiol Dis 2009;35:128-140.

6. Fagan AM, Roe CM, Xiong C, Mintun MA, Morris JC, Holtzman DM. Cerebrospinal fluid tau/beta-amyloid (42) ratio as a prediction of cognitive decline in nondemented older adults. Arch Neurol 2007;64:343-349.

7. Roe CM, Fagan AM, Williams MM, et al. Improving CSF biomarker accuracy in predicting prevalent and incident Alzheimer disease. Neurology 2011;76:501-510.

8. Villemagne VL, Pike KE, Chételat G, et al. Longitudinal assessment of $A \beta$ and cognition in aging and Alzheimer disease. Ann Neurol 2011;69:181-192.

9. Berg L, McKeel DW, Miller JP, et al. Clinicopathologic studies in cognitively healthy aging and Alzheimer diseaserelation of histologic markers to dementia severity, age, sex, and apolipoprotein E genotype. Arch Neurol 1998;55: 326-335.

10. Villareal DT, Grant E, Miller JP, Storandt M, McKeel DW, Morris JC. Clinical outcomes of possible versus probable Alzheimer's disease. Neurology 2003;61: 661-667.

11. Morris JC. The Clinical Dementia Rating (CDR): current version and scoring rules. Neurology 1993;43:2412-2414.

12. Hughes CP, Berg L, Danziger WL, Coben LA, Martin RL. A new clinical scale for the staging of dementia. Br J Psychiatry 1982;140:566-572.

13. Burke WJ, Miller JP, Rubin EH, et al. Reliability of the Washington University Clinical Dementia Rating. Arch Neurol 1988;45:31-32. 
14. Mintun MA, Larossa GN, Sheline YI, et al. [11C]PIB in a nondemented population: potential antecedent marker of Alzheimer disease. Neurology 2006;67:446-452.

15. Fagan AM, Mintun MA, Mach RH, et al. Inverse relation between in vivo amyloid imaging load and cerebrospinal fluid Abeta42 in humans. Ann Neurol 2006;59:512-519.

16. Blennow K, Hampel H, Weiner M, Zetterberg H. Cerebrospinal fluid and plasma biomarkers in Alzheimer disease. Nat Rev Neurol 2010;6:131-144.

17. Gönen M, Heller G. Concordance probability and discriminatory power in proportional hazards regression. Biometrika 2005;92:965-970.

18. Goodglass H, Kaplan E. Boston Diagnostic Aphasia Examination Booklet, 3rd ed. Oral Expression, J. Animal Naming (Fluency in Controlled Association). Philadelphia: Lea \& Febiger; 1983.

19. Armitage SG. An analysis of certain psychological tests used for the evaluation of brain injury. Psychol Monogr 1946;60:48.

20. Grober E, Buschke H, Crystal H, Bang S, Dresner R. Screening for dementia by memory testing. Neurology 1988;38:900-903.

21. Folstein MF, Folstein SE, McHugh PR. "Mini-mental state": a practical method for grading the cognitive state of patients for the clinician. J Psychiatr Res 1975;12:189-198.

22. Johnson DK, Storandt M, Morris JC, Langford ZD, Galvin JE. Cognitive profiles in dementia: Alzheimer disease vs healthy brain aging. Neurology 2008;71:1783-1789.

23. Morris JC, Roe CM, Xiong C, et al. APOE predicts amyloid-beta but not tau Alzheimer pathology in cognitively normal aging. Ann Neurol 2010;67:122-131.

24. Tarawneh R, D’Angelo G, Macy E, et al. Visinin-like protein-1: diagnostic and prognostic biomarker in Alzheimer disease. Ann Neurol 2011;70:274-285.

25. Morris JC, Roe CM, Grant EA, et al. Pittsburgh compound $\mathrm{B}$ imaging and prediction of progression from cognitive normality to symptomatic Alzheimer disease. Arch Neurol 2009;66:1469-1475.

26. Roe CM, Mintun MA, D’Angelo G, Xiong C, Grant EA, Morris JC. Alzheimer disease and cognitive reserve: variation of education effect with carbon 11-labeled Pittsburgh compound B uptake. Arch Neurol 2008;65:1467-1471.

27. Fagan AM, Mintun MA, Shah AR, et al. Cerebrospinal fluid tau and ptau (181) increase with cortical amyloid deposition in cognitively normal individuals: implications for future clinical trials of Alzheimer's disease. EMBO Mo Med 2009;1:371-380.
28. Gönen M. Analyzing Receiver Operating Characteristic Curves with SAS. Cary, NC: SAS Publishing; 2007.

29. Bateman RJ, Xiong C, Benzinger TLS, et al. Clinical and biomarker changes in dominantly inherited Alzheimer's disease. N Engl J Med 2012;367:795-804.

30. Tang MX, Cross $\mathrm{P}$, Andrews $\mathrm{H}$, et al. Incidence of $\mathrm{AD}$ in African-Americans, Caribbean Hispanics, and Caucasians in northern Manhattan. Neurology 2001;56:49-56.

31. Roberts RO, Geda YE, Knopman DS, et al. The incidence of MCI differs by subtype and is higher in men: the Mayo Clinic study of aging. Neurology 2012;78:342-351.

32. Hebert LE, Scherr PA, McCann JJ, Beckett LA, Evans DA. Is the risk of developing Alzheimer's disease greater for women than for men? Am J Epidemiol 2001; 153:132-136.

33. Yip AG, Brayne C, Matthews FE. Risk factors for incident dementia in England and Wales: the Medical Research Council Cognitive Function and Ageing Study. A populationbased nested case-control study. Age Ageing 2006;35: 154-160.

34. Barnes LL, Wilson RS, Bienias JL, Schneider JA, Evans DA, Bennett DA. Sex differences in the clinical manifestations of Alzheimer disease pathology. Arch Gen Psychiatry 2005;62:685-691.

35. Buckner RL, Snyder AZ, Shannon BJ, et al. Molecular, structural, and functional characterization of Alzheimer's disease: evidence for a relationship between default activity, amyloid, and memory. J Neurosci 2005;25:7709-7717.

36. Csernansky JG, Wang L, Swank J, et al. Preclinical detection of Alzheimer's disease: hippocampal shape and volume predict dementia onset in the elderly. Neuroimage 2005;25:783-792.

37. Sheline YI, Raichle ME, Snyder AZ, et al. Amyloid plaques disrupt resting state default mode network connectivity in cognitively normal elderly. Biol Psychiatry 2010; 67:584-587.

38. Cairns NJ, Ikonomovic MD, Benzinger T, et al. Absence of Pittsburgh compound B detection of cerebral amyloid beta in a patient with clinical, cognitive, and cerebrospinal fluid markers of Alzheimer disease. Arch Neurol 2009;66: 1557-1562.

39. Stern Y. Cognitive reserve. Neuropsychologia 2009;47: 2015-2028.

40. Crum RM, Anthony JC, Bassett SS, Folstein MF. Population-based norms for the Mini-Mental State Examination by age and educational level. J Am Med Assoc 1993; 269:2386-2391.

\section{Neurology ${ }^{\circledR}$ Online CME Program}

Earn CME while reading Neurology. This program is available only to online Neurology subscribers. Simply read the articles marked CME, go to www.neurology.org, and click on CME. This will provide all of the information necessary to get started. The American Academy of Neurology (AAN) is accredited by the Accreditation Council for Continuing Medical Education (ACCME) to sponsor continuing medical education for physicians. Neurology is planned and produced in accordance with the ACCME Essentials. For more information, contact AAN Member Services at 800-879-1960. 